\title{
Post traumatic splenic cysts
}

\section{Introduction}

Nonparasitic splenic cysts are uncommon, with only around 800 cases described in the medical literature. Post traumatic splenic cyst forms a part of this entity, and it should be considered if someone has a mass in the left upper abdomen after sustaining abdominal trauma. We report a 17 year old male with history of blunt abdominal trauma and developing a post traumatic splenic cyst.

\section{Case presentation}

Hereby a case report is presented which describes the presentation and management of a case of post traumatic splenic cyst, which was treated with laparotomy and total splenectomy .The procedure, was carried out successfully, with no complication, the patient being discharged home a few days after operation with subsequent follow up.

\section{History}

A17year old male presented to the surgical clinic with complaint of swelling over the left upper abdomen for the past 7 months, gradually increasing in size. The swelling was occasionally painful, there was no history of vomiting, nausea, loss of appetite or fever. There was no definite history of having sustained abdominal trauma.

\section{Physical examination}

The general condition of the patient was fair, a febrile, no pallor, no jaundice not tachypneic.

i. Vital signs were:T 36.7, Pulse:79/min BP:123/78mm Hg.

ii. Chest examination: unremarkable.

iii. Abdominal examination: soft lax abdomen with swelling in the left hypochondrium area extending to the epigastric area and the left lumbar regions. The mass was moving with respiration, nontender, about $12 \times 10 \mathrm{~cm}$. Renal angle was free and no ascites.

iv. Blood tests: WBC 6.500, HGB 12.5, PLT 192000, PT 15.7, PPT 31, INR 1026. Urea and electrolyte, serum amylase, liver function test were all within normal limits (Figure 1).

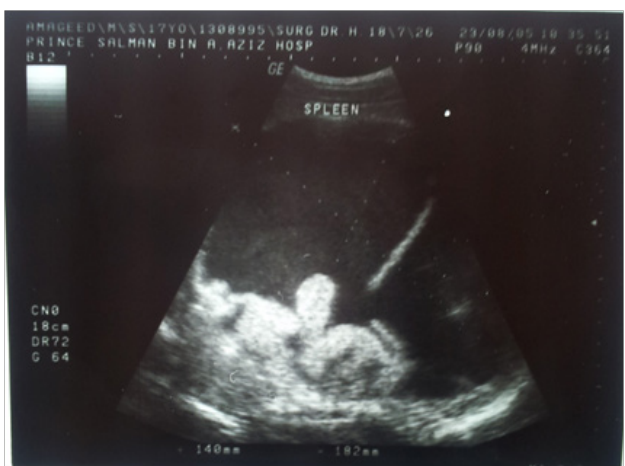

Figure I Ultrasonography of abdomen: revealed a cystic collection juxtaposed to the spleen.
Volume 4 Issue 3 - 2017

\section{Naif AlEnazi, Saud Bin Jadaan, Khaled Ahmad, Arun Bharthuar \\ Department of surgery, Prince Mohammed bin abdulaziz hospital, Saudi Arabia}

Correspondence: Naif AIEnazi, MD, MBA, FICS, FEBS, AF-ACS, Consultant Bariatric and Laparoscopic surgery, Department of surgery, Prince Mohammed bin abdulaziz hospital, P.O.Box 8611 Zip I 1492, Riyadh-Saudi Arabia, Email Surgeon_n@hotmail.com

Received: October 21,2014 | Published: May II, 2017

\section{Radiological examination}

1. X-ray chest: normal lung fields, no pleural effusion, no hemopnemothorax, no fracture ribs, no mediastinal shift.

2. Ultrasonography of abdomen: revealed a cystic collection juxtaposed to the spleen.

The patient was prepared for laparotomy after preoperative anesthetic assessment. Laparotomy was performed through a midline incision and the splenic cyst along with spleen was mobilized and splenectomy performed. The abdomen was closed without a drain. The patient had a smooth postoperative recovery. He received pneumococcal vaccine and was discharged on the fifth day post operative with follow up in the clinic. Histopathological report of the removed cyst revealed a hemorrhagic splenic cyst (Figure 2).

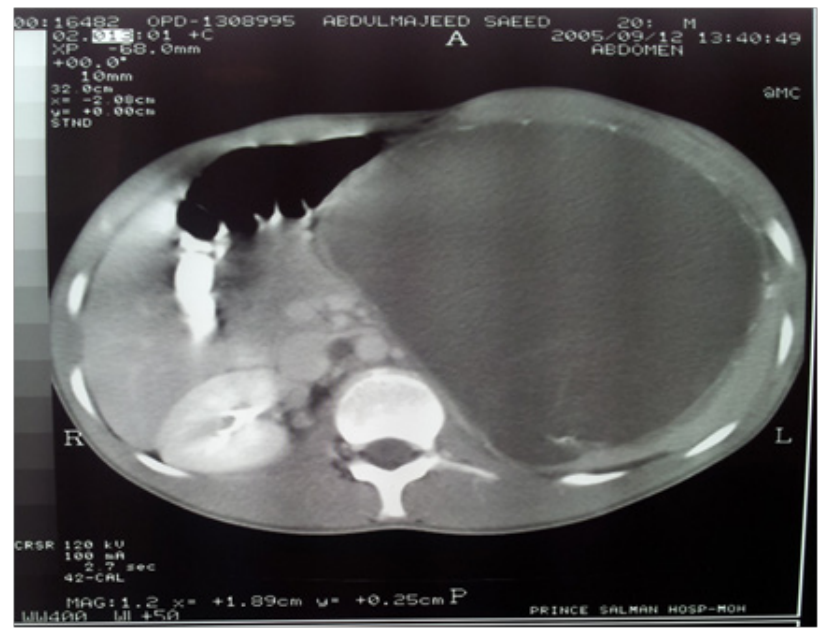

Figure 2 Computerized tomography of the abdomen: confirmed the presence of a splenic cyst of size $|3 \times 1| \mathrm{cm}$. 


\section{Discussion}

Splenic cysts are classified as primary (true cyst ), which include parasitic and non parasitic cysts, and as secondary (pseudo cyst) according to their etiology and pathophysiology. ${ }^{1,2}$ True cysts have an epithelial or mesothelial lining, on the other hand, pseudocysts have no cellular lining, only a fibrous capsule. ${ }^{3-5}$ A vast majority of splenic cysts are pseudo cysts resulting from blunt trauma. Other causes include infarction or infection. ${ }^{4}$ True non-parasitic splenic cysts are a rare pathology represented by some 800 cases recorded worldwide. The method of choice for the treatment of this condition is surgical intervention. Today, open surgery is substituted by minimal invasive methods, such as laparoscopic splenectomy. ${ }^{5}$ Treatment decisions are often based on the size of the cyst and related symptoms. Small, asymptomatic cysts are best followed with serial US or CT because spontaneous resolution o traumatic pseudocyst can occur. Though the risk of major complications is small, larger cysts and those that are symptomatic should be treated surgically. Less invasive procedures, such as aspiration, may be associated with cyst recurrence. ${ }^{6}$ Traditional surgical management of splenic cyst has been either partial or total splenectomy. However, splenectomy places the patient at risk for development of postsplenectomy sepsis..$^{3-5,7}$ Splenic cysts are usually diagnosed incidentally because of the lack of typical clinical symptoms. Complaints of the patients are generally related to mass effect of the cyst, which causes compression of neighbouring organs. Large cysts may cause abdominal pain, early satiety, nausea, vomiting, constipation, or hydronephrosis. In the physical examination, a mass with smooth surface is usually detected in the left hypochondrium especially if the cyst size is large. All patients with a left hypochondrial mass should be interrogated if there is any remote history of trauma. Masses of the spleen are uncommon lesions, and these can be summarized as: congenital cysts, inflammatory masses, vascular masses, posttraumatic masses, benign neoplastic masses, malignant neoplastic masses. In addition, other causes of splenomegaly like, myeloid metaplasia, mononucleosis, haemolytic anemia, and portal hypertension must be excluded. ${ }^{7,8-10}$

Diagnosis of splenic cyst is generally based on radiological studies. Ultrasonography and CT imaging may help to distinguish cystic from solid lesions. These imaging modalities are also helpful in preoperatively evaluating whether the cyst is multi or unilocular, its location in the spleen and its relation to the surrounding structures. However, because of the similarity in Ultrasound and CT findings of primary and secondary splenic cyst there may be a diagnostic confusion. Nonparasitic cysts of the spleen are often treated wrongly as echinococcal cysts and in some cases specific medical treatment has been prescribed. Although some authors have suggested we think that, MRI may be helpful in this situation as in our case. ${ }^{11-14}$

\section{Conclusion}

Splenic cyst one of the cases that can be developed after the abdominal trauma which needs to be looked carefully and not to be missed.

\section{Acknowledgements}

None.

\section{Conflict of interest}

The author declares no conflict of interest.

\section{References}

1. Dimitrios V Avgerinos. Post-traumatic Splenic Cyst treated with laparoscopy: two case report. Cases J. 2009;2:7976.

2. Ieva Martinaityte. Post-traumatic splenic pseudocyst: case report and review of the literature, LIETUVOS CHIRURGIJA. Lithuanian Surgery. 2009;7(3-4):69-73.

3. Labruzzo C, Haritopoulos KN, El Tayar AR, et al. Post-traumatic cyst of spleen: a case report and review of the literateure. Int Surg. 2002;87(3):152-156

4. Wu HM, Kortbeek JB. Management of spleenic pseudocysts following trauma: a retrospective case series. Am J Surg. 2006;191(5):631-634.

5. Chawla Lt Col S, Kumar BP, et al. post-traumatic pseudocyst of the spleen. MJAFI. 2005;61:279-280.

6. Murat Acar. Posttraumatic splenic cyst diagnostic value of MRI. Eur J Gen med. 2008;5(4):242-244.

7. Seshadri PA, Poenaru D, Park A. Laproscopic splenic cystectomy: a case report. J Pediatr Surg. 1998;33(9):1439-1440.

8. Van der Zee DC, Kramer WL, Ure BM, et al. Laproscopic management of a large posttraumatic splenic cyst in a child. Surg Endosc. 1999;13(12):12411242.

9. Targarona EM, Martinez J, Ramos C, et al. Conservative laproscopic treatment of a posttrumatic splenic cyst. Surg Endosc. 1995;9(1):71-72.

10. Calligaris L, Bortul M. Laproscopic treatment of a nonparasitic splenic cyst, case report. J Laparoendosc Surg. 1996;6(6):431-434.

11. Mclean AL, Broussard E, McCarter MD. laparoscopic marsupialization of nonparasitic splenic cysts. Contemporary Surg. 2000;56:541-545.

12. Souka H, Loosemore T, Taylor RS. Laparoscopic partial cystectomy for post-traumatic splenic pseudocyst. Endoscopy. 1997;29(7):S41.

13. Linhares MM, Caetano EM, Louzano R, et al. Surgical resection of a traumatic spleen cyst by laparoscopy. Int Surg. 1998;83(4):308-310.

14. Lang J, Kostin R. Traumatic rupture of a splenic cyst. Surg Rounds. $2000 ; 23: 442-457$. 